\title{
On converse duality in complex nonlinear programming
}

\section{J. Parida}

In this note a converse duality theorem is proved for a class of nonlinear programming problems over polyhedral cones in finite dimensional complex space by a direct use of a Kuhn-Tucker type necessary and sufficient condition for constrained optimization in complex space.

\section{Introduction}

Abrams and Ben-Israel [2] and Abrams [1] have established a converse duality theorem for nonlinear programming in complex space. For the proof they have assumed the existence of an auxiliary analytic function. Craven and Mond [5] have given a converse duality theorem for complex space through a method of proof that depends on mapping a complex minimization problem onto an equivalent minimization problem in real space. In [2], Abrams and Ben-Israel have obtained a necessary condition of the KuhnTucker type for a class of nonlinear programming problems in complex space. In the present paper, we have modified their result so as to be applicable to the case where there may occur equality constraints in the constraint set and then used this modified result to prove a converse duality theorem for complex space.

\section{Notations and preliminaries}

Let $C^{n}\left(R^{n}\right)$ denote the $n$-dimensional complex (real) space, with hermitian (euclidean) norm. $R_{+}$denotes the half line $[0, \infty)$. If $A$ is

Received 2 September 1975. 
a complex matrix or vector, then $A^{T}, \bar{A}$ and $A^{H}$ denote its transpose, complex conjugate, and conjugate transpose. $S \subset c^{m}$ is a polyhedral cone if it is finite intersection of closed half-spaces in $C^{m}$, each containing 0 on its boundary. The polar $S^{*}$ of $S$ is defined by

$$
S^{*}=\left\{y \in C^{m}: x \in S \Rightarrow \operatorname{Re} y^{H} x \geq 0\right\} \text {. }
$$

We shall make use of the following [1], [2], [5]: if $S$ and $T$ are polyhedral cones in $c^{m}$, then

$$
(S \times T)^{*}=S^{*} \times T^{*},
$$

$S \times T$ is a polyhedral cone.

If $S=C^{m}$, then $S^{*}=\{0\}$.

Define the linear manifold $Q \subset C^{2 n}=C^{n} \times C^{n}$ by

$$
Q=\left\{\left(w^{1}, w^{2}\right) \in C^{2 n}: w^{2}=\overline{w^{I}}\right\} \text {. }
$$

The analytic function $f: Q \rightarrow C$ has a convex real part with respect to $R_{+}$on $Q$ if for all $z^{1}, z^{2} \in Q$,

$$
\operatorname{Re}\left[f\left(z^{1}, \overline{z^{1}}\right)-f\left(z^{2}, \overline{z^{2}}\right)-\nabla_{z}^{T} f\left(z^{2}, \overline{z^{2}}\right)\left(z^{1}-z^{2}\right)-\nabla \frac{T}{z} f\left(z^{2}, \overline{z^{2}}\right)\left(\overline{z^{1}}-\overline{z^{2}}\right)\right] \geq 0,
$$

where $\nabla_{z} f\left(z^{2}, \overline{z^{2}}\right)$ and $\nabla-f f\left(z^{2}, \overline{z^{2}}\right)$ denote, respectively, the column vectors of partial derivatives

$$
\frac{\partial f\left(z^{2}, \overline{z^{2}}\right)}{\partial w_{i}^{1}} \text { and } \frac{\partial f\left(z^{2}, \overline{z^{2}}\right)}{\partial w_{i}^{2}}
$$

The analytic function $g: Q \rightarrow C^{m}$ is concave with respect to $S$ on $Q$ if for all $z^{1}, z^{2} \in Q$,

$$
D_{z} g\left(z^{2}, \overline{z^{2}}\right)\left(z^{1}-z^{2}\right)+D_{z^{g}}\left(z^{2}, \overline{z^{2}}\right)\left(\overline{z^{1}}-\overline{z^{2}}\right)+g\left(z^{2}, \overline{z^{2}}\right)-g\left(z^{1}, \overline{z^{1}}\right) \in S
$$

where $D_{z} g\left(z^{2}, \overline{z^{2}}\right)$ and $D_{z} g\left(z^{2}, \overline{z^{2}}\right)$ denote, respectively, the $m \times m$ matrices whose $i, j-t h$ elements are 


$$
\frac{\partial g_{i}\left(z^{2}, \overline{z^{2}}\right)}{\partial w_{j}^{1}} \text { and } \frac{\partial g_{i}\left(z^{2}, \overline{z^{2}}\right)}{\partial w_{j}^{2}}
$$

If $f: Q \rightarrow C, f^{R}: Q \rightarrow R$, and $f^{I}: Q \rightarrow R$ are analytic on $Q$, and $f(z, \bar{z})=f^{R}(z, \bar{z})+i f^{I}(z, \bar{z})$ then

$$
\overline{\nabla_{z} f(z, \bar{z})}+\nabla-f(z, \bar{z})=2 \nabla-f^{R}(z, \bar{z})
$$

If $f: Q \rightarrow C$ is an analytic function, then (see [5], p. 619)

$$
\left(\frac{\partial f}{\partial w_{i}^{I}}\right]=\frac{\partial \bar{f}}{\partial w_{i}^{2}} \text { and }\left(\frac{\partial f}{\partial w_{i}^{2}}\right)=\frac{\partial \bar{f}}{\partial w_{i}^{I}} \text {. }
$$

\section{Complex version of the Kuhn-Tucker conditions}

We shall make use of the following results of Abrams and Ben-Israel [2].

THEOREM 2.1. Let (P) denote the problem

$$
\begin{array}{ll}
\text { MINIMIZE } & \operatorname{Re} f(z, \bar{z}) \\
\text { SUBJECT TO } & g(z, \bar{z}) \in S
\end{array}
$$

where $S$ is a polyhedral cone in $C^{m}$, and $f: Q \rightarrow C, g: Q \rightarrow C^{m}$ are analytic in a neighborhood of a qualified point $\left(z^{0}, \overline{z^{0}}\right)$. A necessary condition for $\left(z^{0}, \overline{z^{0}}\right)$ to be optimal is that there exists a $u \in S^{*}$ such that

$$
\operatorname{Re} u^{H} g\left(z^{0}, \overline{z^{0}}\right)=0
$$

and

$$
\overline{\nabla_{z} f\left(z^{0}, \overline{z^{0}}\right)}+\nabla_{z} f\left(z^{0}, \overline{z^{0}}\right)=D_{z^{H}} g\left(z^{0}, \overline{z^{0}}\right) u+D \frac{T}{z^{\prime}} g\left(z^{0}, \overline{z^{0}}\right) \bar{u} .
$$

REMARKS 2.2. A point $\left(z^{0}, \overline{z^{0}}\right)$ qualified means it is a feasible point of $(P)$ and the (Kuhn-Tucker) constraint qualification defined in [3] holds at this point.

If $f: Q \rightarrow C$ is analytic and has convex real part with respect to $R_{+}$on $Q$ and $g: Q \rightarrow C^{m}$ is analytic and concave with respect to $S$ on 
$Q$, then in Theorem 2.1 the necessary condition is also sufficient [1].

We want to modify Theorem 2.I so as to include equality, as well as inequality, constraints.

COROLLARY 2.3. If (P) itsel $\hat{f}$ is of the form

$$
\begin{array}{ll}
\text { MINIMIZE } & \operatorname{Re} f(z, \bar{z}, w, \bar{w}) \\
\text { SUBJECT TO } & G(z, \bar{z}, w, \bar{w}) \in S=
\end{array}
$$

$$
H(z, \bar{z}, w, \bar{w})=0, w \in T,
$$

then a necessary condition for $\zeta=\left(z^{0}, \overline{z^{0}}, w^{0}, \overline{w^{0}}\right)$ to be optimal is $u \in S^{*}$,

(2.4) $\overline{\nabla_{w} f(\zeta)}+\nabla-f(\zeta)-D_{w}^{H} G(\zeta) u-D \frac{T}{w} G(\zeta) \bar{u}-D_{w}^{H} H(\zeta) v-D \frac{T}{w}(\zeta) \bar{v} \epsilon T^{*}$,

$$
\operatorname{Re} u^{H} G(\zeta)=0 \text {, }
$$

(2.6) $\operatorname{Re}\left(\nabla_{w}^{T} f(\zeta)+\nabla \frac{H}{w} f(\zeta)-u^{H} D_{w} G(\zeta)-u^{T} \overline{D_{w} G(\zeta)}-v^{H} D_{w} H(\zeta)-v^{T} \overline{D_{w}^{H(}(\zeta)}\right) w^{0}=0$,

and

$$
\overline{\nabla_{z} f(\zeta)}+\nabla \frac{-}{z} f(\zeta)=D_{z}^{H} G(\zeta) u+D_{z}^{T} G(\zeta) \bar{u}+D_{z}^{H} H(\zeta) v+D_{z}^{\frac{T}{z}} H(\zeta) \bar{v} .
$$

Proof. The constraint set (2.3) can be written as

$$
\left\{\begin{array}{c}
G(z, \bar{z}, w, \bar{w}) \\
H(z, \bar{z}, w, \bar{w}) \\
w
\end{array}\right\} \in S \times\{0\} \times T .
$$

An easy application of Theorem 2.1 then gives the required result.

\section{Converse duality}

Assume $f(z, \bar{z})$ and $g(z, \bar{z})$ in (P) satisfy the hypothesis of Theorem 2.1. A dual of (P) in the sense of Wolfe [7] is

(D) MAXIMIZE $\operatorname{Re} f(x, \bar{x})-\operatorname{Re} y^{H} g(x, \bar{x})$

SUBJECT то $\psi(x, \bar{x}, y, \bar{y})=\overline{\nabla_{z} f(x, \bar{x})}+\nabla_{z} f(x, \bar{x})-D_{z}^{H} g(x, \bar{x}) y$

$$
-D \frac{T}{z} g(x, \bar{x}) \bar{y}=0, y \in S^{*} \text {. }
$$

THEOREM 3.1. Let $f: Q \rightarrow C$ be analytic and have convex real part 
with respect to $R_{+}$on $Q$, let $S$ be a polyhedral cone in $c^{m}$, and let $g: Q \rightarrow C^{m}$ be analytic and concave with respect to $S$ on $Q$. If $\left(z^{0}, \overline{z^{0}}, \overline{u^{0}}\right)$ is an optimal solution of (D) such that the matrix

$$
\left[\begin{array}{ll}
D \frac{H}{z} \psi(x, \bar{x}, y, \bar{y}) & D_{z}^{T} \psi(x, \bar{x}, y, \bar{y}) \\
D_{z}^{H} \psi(x, \bar{x}, y, \bar{y}) & D_{z}^{T} \psi(x, \bar{x}, y, \bar{y})
\end{array}\right]
$$

is nonsingular at $\left(z^{0}, \overline{z^{0}}, u^{0}, \overline{u^{0}}\right)$, then $\left(z^{0}, \overline{z^{0}}\right)$ is an optimal solution of $(P)$.

Proof. We shall suppose that $\left(z^{0}, \overline{z^{0}}, \overline{u^{0}}\right)$ is a qualified point relative to the feasible region of (D). So $\left(z^{0}, \overline{z^{0}}, \overline{u^{0}}\right)$ is a feasible point of (D) and we have

$$
\overline{\nabla_{z} f\left(z^{0}, \overline{z^{0}}\right)}+\nabla \frac{-f}{z}\left(z^{0}, \overline{z^{0}}\right)-D_{z^{H}}^{H}\left(z^{0}, \overline{z^{0}}\right) u^{0}-D \frac{T}{z} g\left(z^{0}, \overline{z^{0}}\right) \overline{u^{0}}=0
$$

and

$$
u^{0} \in S^{*}
$$

Utilising Corollary 2.3, we get

$$
\begin{gathered}
g\left(z^{0}, \overline{z^{0}}\right)+D_{z^{g}}\left(z^{0}, \overline{z^{0}}\right) v+D-g\left(z^{0}, \overline{z^{0}}\right) \bar{v} \in S, \\
\operatorname{Re} u^{0^{H}}\left\{g\left(z^{0}, \overline{z^{0}}\right)+D_{z^{\prime}} g\left(z^{0}, \overline{z^{0}}\right) v+D-g\left(z^{0}, \overline{z^{0}}\right) \bar{v}\right\}=0,
\end{gathered}
$$

and

$$
D_{z}^{H} \psi\left(z^{0}, \overline{z^{0}}, u^{0}, \overline{u^{0}}\right) v+D \frac{T}{z} \psi\left(z^{0}, \overline{z^{0}}, u^{0}, \overline{u^{0}}\right) \bar{v}=0
$$

Combining (3.6) with its complex conjugate, we obtain

$$
\left[\begin{array}{cc}
D \frac{H}{z} \psi\left(z^{0}, \overline{z^{0}}, u^{0}, \overline{u^{0}}\right) & D_{z}^{T} \psi\left(z^{0}, \overline{z^{0}}, u^{0}, \overline{u^{0}}\right) \\
D_{z}^{H} \psi\left(z^{0}, \overline{z^{0}}, u^{0}, \overline{u^{0}}\right) & D_{z}^{T} \psi\left(z^{0}, \overline{z^{0}}, u^{0}, \overline{u^{0}}\right)
\end{array}\right]\left[\begin{array}{l}
v \\
\bar{v}
\end{array}\right]=0 .
$$

The second part of the statement of Theorem 3.1 assures us that the 
solution $(v, \bar{v})$ of the homogeneous system (3.7) is unique, and therefore

$$
v=0 \text {. }
$$

Using (3.8) we find that (3.4) and (3.5) become

$$
g\left(z^{0}, \overline{z^{0}}\right) \in S
$$

and

$$
\operatorname{Re} u^{0^{H}} g\left(z^{0}, \overline{z^{0}}\right)=0 \text {. }
$$

Equation (3.9) implies that $\left(z^{0}, \overline{z^{0}}\right)$ is feasible for (P). Since Re $f$ is convex with respect to $R_{+}$on $Q$ and $g$ is concave with respect to $S$ on $Q$, the conditions (3.2), (3.3), and (3.10) are sufficient for optimality, and thus $\left(z^{0}, \overline{z^{0}}\right)$ is an optimal solution for $(\mathrm{P})$.

REMARKS 3.2. Let $\varphi=f^{R}(z, \bar{z})$ and let partial derivatives be denoted by subscripts. Using identities $(A)$ and $(B)$, the matrix (3.1) becomes

$$
2\left[\begin{array}{ll}
\varphi_{z z} & \varphi_{\bar{z} z} \\
\varphi_{z \bar{z}} & \varphi_{\bar{z} z}
\end{array}\right]-\left[\begin{array}{ll}
g_{z z} & g_{\bar{z} z} \\
g_{z \bar{z}} & g_{\overline{z z}}
\end{array}\right] \bar{y}-\left[\begin{array}{cc}
\bar{g}_{z z} & \bar{g}_{\bar{z} z} \\
\bar{g}_{z \bar{z}} & \bar{g}_{\overline{z z}}
\end{array}\right] y,
$$

which is similar to the matrix obtained in [5] (Theorem 1, p. 622).

Abrams in [1] (Theorem 4, p. 627) has assumed the existence of an analytic function $\alpha: c^{m} \rightarrow c^{n}$ with certain properties. It can be checked that for the existence of such a function, non-singularity of the matrix (3.1) is a sufficient condition (see [4], Theorem 9, p. 39).

One can prove this theorem by using Fritz John type necessary conditions [6], which requires no constraint qualifications, thus being able to eliminate the assumption that the optimal solution of the dual is a qualified point.

\section{References}

[1] Robert A. Abrams, "Nonlinear programming in complex space: sufficient conditions and duality", J. Math. Anal. Appl. 38 (1972), 619-632. 
[2] Robert A. Abrams and Adi Ben-Israel, "Nonlinear programming in complex space: necessary conditions", SIAM J. Control 9 (1971), 606-620.

[3] Robert A. Abrams, Adi Ben-Israel, "Complex mathematical programming" (Report No. 6y-11, Northwestern University, Evanston, Illinois, 1969).

[4] Salomon Bochner and William Ted Martin, Several complex variables (Princeton Mathematical Series, 10. Princeton University Press, Princeton, 1948).

[5] B.D. Craven and B. Mond, "Converse and symmetric duality in complex nonlinear programming", J. Math. Anal. Appl. 37 (1972), 617-626.

[6] B.D. Craven and B. Mond, "Real and complex Fritz John theorems", $J$. Math. Anal. Appl. 44 (1973), 773-778.

[7] P. Wolfe, "A duality theorem for non-linear programming", Quart. Appl. Math. 19 (1961), 239-244.

Department of Mathematics,

Regional Engineering College,

Rourkela,

Orissa,

India. 